\title{
Site-specific Protein Photochemical Covalent Attachment to Carbon Nanotube Side Walls and its Electronic Impact on Single Molecule Function.
}

Suzanne K. Thomas ${ }^{\dagger a}$, W. David Jamieson ${ }^{\$ a}$, Rebecca E.A. Gwyther ${ }^{\ddagger a}$, Ben J, Bowen ${ }^{\ddagger a}$, Adam Beachey ${ }^{\dagger}$, Harley L. Worthy ${ }^{\ddagger}$, J. Emyr Macdonald ${ }^{\dagger}$, Martin Elliott ${ }^{\dagger *}$, Oliver K Castell ${ }^{\$ \star}$, \& D. Dafydd Jones ${ }^{\ddagger *}$.

$\uparrow$ School of Physics and Astronomy, Cardiff University, Cardiff, UK. CF24 3AA.

\$ School of Biosciences, Cardiff University, Cardiff, UK. CF10 3AX

\$ School of Pharmacy and Pharmaceutical Sciences, Cardiff University, Cardiff, UK. CF10 3NB

a These authors contributed equally to the work

* Corresponding authors:

Dafydd Jones, School of Biosciences, Sir Martin Evans Building, Cardiff University, Cardiff, CF10 3AX, UK. Email: jonesdd@cardiff.ac.uk

Oliver Castell, School of Pharmacy and Pharmaceutical Sciences, Redwood Building, Cardiff University, Cardiff, CF10 3NB, UK. Email: CastellO@cardiff.ac.uk

Martin Elliott, School of Physics and Astronomy, Queen's Building, Cardiff University, Cardiff, CF24 3AA, UK. Email. martin.elliott@astro.cf.ac.uk

Current address: SKT, College of Engineering, Swansea University, UK; HLW, Biocatalysis Centre, University of Exeter, Exeter, UK. 


\section{ToC}

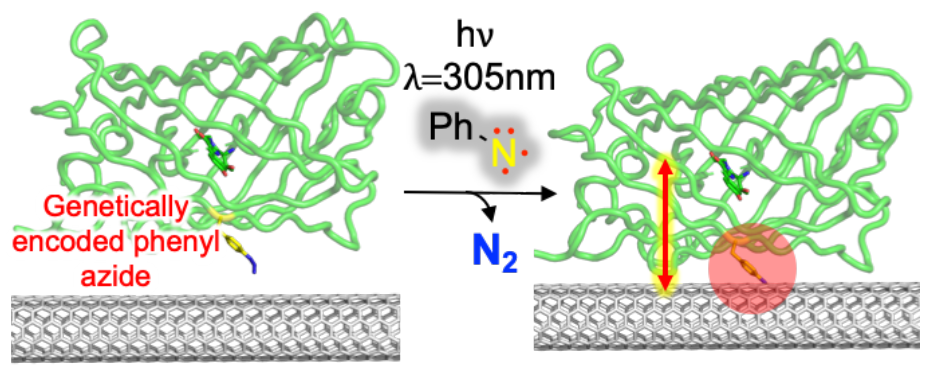

\section{Abstract}

Functional integration of proteins with carbon-based nanomaterials such as nanotubes holds great promise in emerging electronic and optoelectronic applications. Control over protein attachment poses a major challenge for consistent and useful device fabrication, especially when utilizing single/few molecule properties. Here, we exploit genetically encoded phenyl azide photochemistry to define the direct covalent attachment of four different proteins, including the fluorescent protein GFP and a $\beta$-lactamase binding protein (BBP), to carbon nanotube side walls. AFM showed that on attachment BBP could still recognize and bind additional protein components. Single molecule fluorescence revealed that on attachment to SWCNTs function was retained and there was feedback to GFP in terms of fluorescence intensity and improved resistance to photobleaching; GFP is fluorescent for much longer on attachment. The site of attachment proved important in terms of electronic impact on GFP function, with the attachment site furthest from the chromophore having the larger effect on fluorescence. Our approach provides a versatile and general method for generating intimate protein-CNT hybrid bioconjugates. It can be potentially applied easily to any protein of choice; attachment position and thus interface characteristics with the CNT can easily be changed by simply placing the phenyl azide chemistry at different residues by gene mutagenesis. Thus, our approach will allow consistent construction and modulate functional coupling through changing the protein attachment position.

Keywords. Bionanohybrids; site-specific protein conjugation; phenyl azide; expanded genetic code; carbon nanotubes; single molecule fluorescence 
Here we present a general facile approach to directly and intimately covalently attach proteins to pristine carbon nanotubes (CNT) sidewalls at defined residues using light and assess how such intimate coupling influences single molecule protein function. The functionalisation of nanoscale conducting materials such as graphene and CNTs with biomolecules is an important step in the development of molecular electronic components and miniaturised bio/chemical sensors (1-3). The conductance and optical properties of single walled CNTs (SWCNTs)(4) are very sensitive to their local environment through perturbation of the pi electron network; functionalization (5-7) and associated secondary molecular events (e.g. binding, catalysis, energy transfer) can modulate nanotube properties and so introduce new and useful characteristics to pristine CNTs $(1,2,8)$. The cylindrical nature of CNTs arguably improve their biocompatibility compared to planer graphene as the reduced hydrophobic surface area together with comparable diameters minimises disruption of the biomolecule, through for example induced protein unfolding. CNTs also allows for the construction of defined nanowires across electrodes $(9,10)$. Due to their inherent molecular function and exquisite recognition properties, protein molecules have become a particular focus for constructing hybrid CNT nanomaterials, both in terms of assembling new devices and understanding fundamentals of dynamical biological function at the individual molecule level $(1,3,11-14)$.

Given the importance to constructing biohybrid CNTs, protein attachment approaches has been the focus of many studies (see $(5,8,15,16)$ for recent reviews); despite various different approaches being developed to facilitate proteinCNT interfacing limitations persist such as consistent, stable and defined interfacing during the bioconjugation fabrication step. Robust, defined, homogenous and intimate attachment between the protein and the CNT (17) will facilitate optimal and maximal communication between the two components. Another requirement is to understand how generating such an intimate interface affects protein function, especially with regards to the residue on the protein that acts as the attachment site. The problem is that the interface residue with respect to the protein is rarely defined leading to an array of different attachment positions and thus protein-CNT interface environments. While heterogenous protein attachment by traditional approaches such as amine functionalisation (via lysine residues commonly found on a proteins surface) may not considered a problem for bulk ensemble measurements and applications where signal averaging takes place, it becomes a significant problem 
when single or few molecules are to be utilised: different attachment sites can give rise to heterogenous and inconsistent output. In the most drastic of cases, attachment may remove protein function through, for example, blocking active sites or causing disruptive conformation changes. Furthermore, many approaches for functionalising CNTs with proteins require the CNTs to be chemically oxidised to introduce the required reactive groups (usually hydroxyl and carboxyl) (See $(1,6)$ for recent reviews), which is difficult to control, leading to wide-scale disruption of $\mathrm{sp}^{2}$ networks that give CNTs their useful inherent properties, such as conductance. Adaptor molecules may also be required that can increase the spacing between protein and CNT surface so reducing intimacy and thus sensitivity. Here we seek to address the current issues with protein-CNT interfacing by utilising phenyl azide photochemistry $(18,19)$ that can be incorporated at effectively any selected residues in a recombinantly produced target protein of choice using a reprogrammed genetic code (Figure 1a) (20-23). Irradiation with near UV light results in the loss of molecular nitrogen, generating a reactive nitrene radical that can attack electron rich regions (24-26), such as that present in the CNT side wall (see Supporting Scheme S1). Phenyl azide (though incorporation of the non-canonical amino acid azF; Figure 1) when embedded in a protein shows similar photochemistry to small molecule organic phenyl azides $(21,27-30)$ and has been shown to facilitate bulk attachment to $\mathrm{sp}^{2}$ rich flat planer materials such as graphene to generate monolayers (31). As mentioned above, the cylindrical shape and dimensions make CNTs potentially more useful for protein attachment than planer graphene. We demonstrate the broad applicability of the method by attaching four distinct proteins to CNTs. We also show how attachment of GFP at two different positions influences fluorescence at the single molecule level, which demonstrates how such intimate interactions manifest at the protein level and how different attachment positions can elicit distinct effects. 


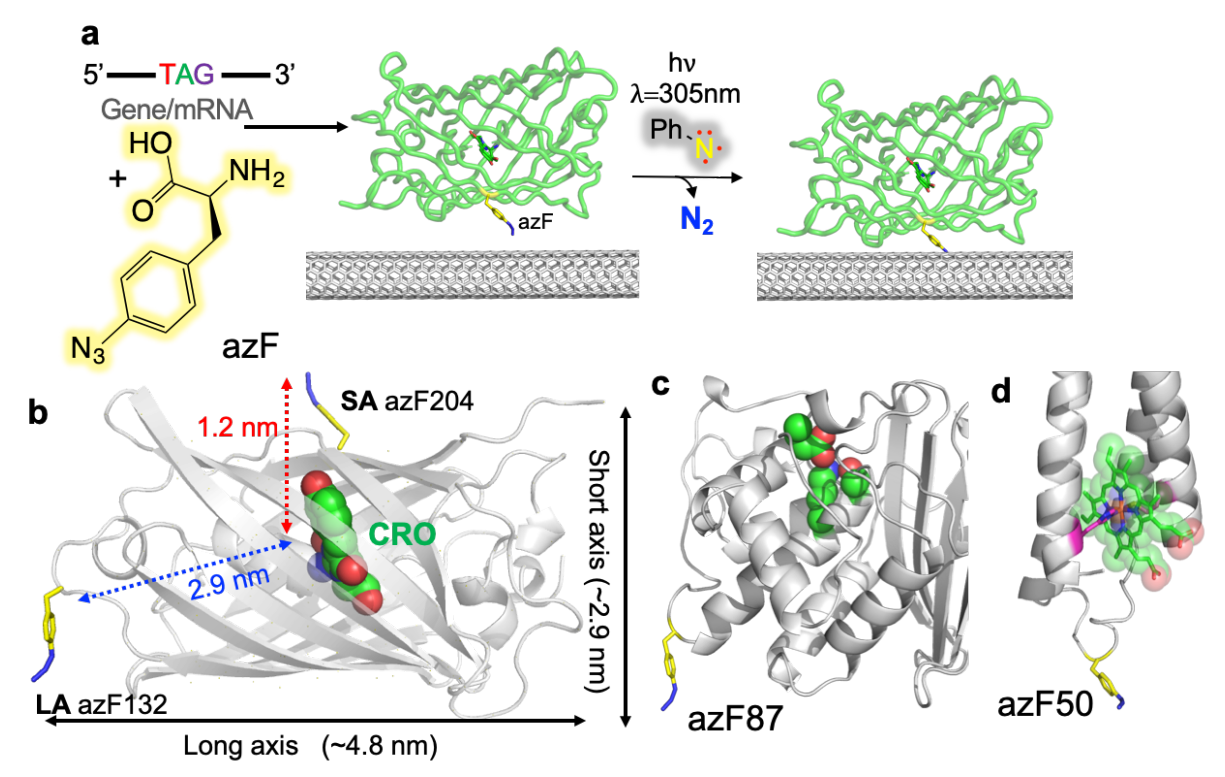

Figure 1. Direct protein attachment to CNTs via phenyl azide photochemistry. (a) Scheme of photochemical insertion of a protein via genetically encoded phenyl azide chemistry (a more detailed chemical scheme is shown in Supporting Scheme S1). (b) Structure and placement of azF in the superfolder version of GFP $(32,33)$. AzF residues are coloured yellow and annotated. The chromophore (CRO) is shown as green spheres. In (b), the different axes in terms of length (long; LA) and breadth (short; SA) of each protein are also shown. Structure and placement of azF in two additional proteins: (c) the $\beta$-lactamase TEM (BL) at residue 87 (mutation Gly87azF) with active site residue shown in green; (d) cytochrome $b_{562}$ at residue 50 (mutation Asp50azF), with the haem centre (shown in green). The azF mutations (yellow sticks) where computationally modelled as outlined in the Supporting Methods.

\section{Results and discussion}

Residue specific attachment of GFP to SWCNTs side-walls. Autofluorescent proteins such as green fluorescent protein (GFP; Figure 1b) are of current interest in the nanosciences as they are finding uses outside classical cell biology in areas ranging from optically gated transistors (34), LEDs through stimulated emission (35, 36) and as light capture and energy transfer devices (37), such as for use in solar cell systems. They have particular advantages over organic molecules, not least because of their eco-friendly nature but that the protein encases the chromophore protecting it from the external environment and allowing modulation of its electronic 
properties through engineered changes in coupled bond networks. The protein also provides the chemical framework for facilitating light induced long range charge transfer, a critical process for fluorescence that is thought to occur by distinct pathways (38-40). Thus, GFP's use in the nanosciences would be facilitated by the ability to couple it in a site-specific manner to electronically active materials such as CNTs.

Light-facilitated attachment of functional proteins to SWCNTs was initially demonstrated using azF variants of the superfolder version of GFP (Figure 1b) (32, 40-43). The two mutants sample different configurations with respect to the $\beta$-barrel structure, distances from the functional centre (the chromophore), and different potential charge transfer pathways (vide infra). Two of the mutants are termed short axis (GFPSA; mutation Q204azF) and long axis (GFPLA; mutation E132azF). The mutants have been characterised previously (33), with differential effects on fluorescence observed once attached via Click chemistry in a 1-to-1 manner to the end-walls of DNA wrapped CNTs (41). Here, we will examine the impact on fluorescence through photochemical attachment to the side wall of pristine CNTs.

On exposure to $305 \mathrm{~nm}$ light (intensity $18 \mathrm{Wm}^{-2}$ ) followed by washing, discrete spots were observed along the length of the SWCNTs with heights consistently higher ( 1$1.5 \mathrm{~nm}$ ) than that of the elongated tube structure (Figures 2, S1 and S2). This indicates that the engineered GFP proteins bound strongly to the SWCNTs indicative of covalent attachment, which is confirmed by control experiments. Single molecule fluorescence confirmed that GFP was still functional on attachment (vida infra). GFP fluorescence is dependent on the protein being correctly folded (44). Thus, attachment has not resulted in the protein unfolding. In the absence of UV treatment, there were no observed discrete spots observed by AFM imaging after the coverslip had been washed highlighting the importance of UV to generating the reactive phenyl nitrene radical required to covalent linkage (Figure 2). Furthermore, no discrete spots were observed when the wild-type GFP (GFPWT; no azF present) was used, including after irradiation (Figure S3) confirming the importance of the azF residue for permanent attachment. AFM imaging of GFPWT prior to rinsing shows the extent of protein coverage and thus the effectiveness of the subsequent washing procedure (Figure S3c). 
The measured heights of the proteins in air are lower than expected based on the simple addition of the nanotube height plus the protein height (based on the crystallographic information highlighted in the Figure 1). In reality, it is unlikely that such an arrangement will occur and our modelling of GFP with the introduced azF residue suggests this simple assumption is unlikely to be the case; the proteins could attach along the available circumference of the tube and not just protrude from the apex (relative to the coverslip surface) of the tube. The proteins may be positioned towards the sides of the nanotubes (relative to the surface) so reducing overall apparent height compared to the CNT (see Figures $2 \mathrm{~d}$ and $2 \mathrm{~g}$ for schematic representation). Furthermore, AFM is known to underestimate heights of soft matter such as biomolecules $(45,46)$. The SWCNTs were also measured to be around 0.5$1 \mathrm{~nm}$ in height, which is smaller than the $0.8-1.5 \mathrm{~nm}$ diameter range of the tubes (Figures 2c, 2f, S1 and S2). The overall increase in width of the protein spots compared to CNTs is an also important independent validation of protein binding. Further evidence for permanent attachment is provided below in the single molecule fluorescence analysis (vide infra). When looking at the disruption of proteins along the length of the tubes, $10 \mathrm{nM}$ routinely generated 2 proteins per $100 \mathrm{~nm}$ of nanotube for GFPSA. GFPLA varied between 1-2 proteins per $100 \mathrm{~nm}$. Increasing the protein concentration of GFPSA to $100 \mathrm{nM}$ still results in a distribution of 2 proteins per $100 \mathrm{~nm}$. AFM imaging suggests that $100 \mathrm{nM}$ of GFP saturates the surface prior to photoactivation and rinsing (Figure S3b). Thus, the observed distribution may reflect initial different regions of the protein adsorbing to the SWCNT, with only those proteins whose azF residue is close to the SWCNT covalently crosslink on illumination with UV light. 


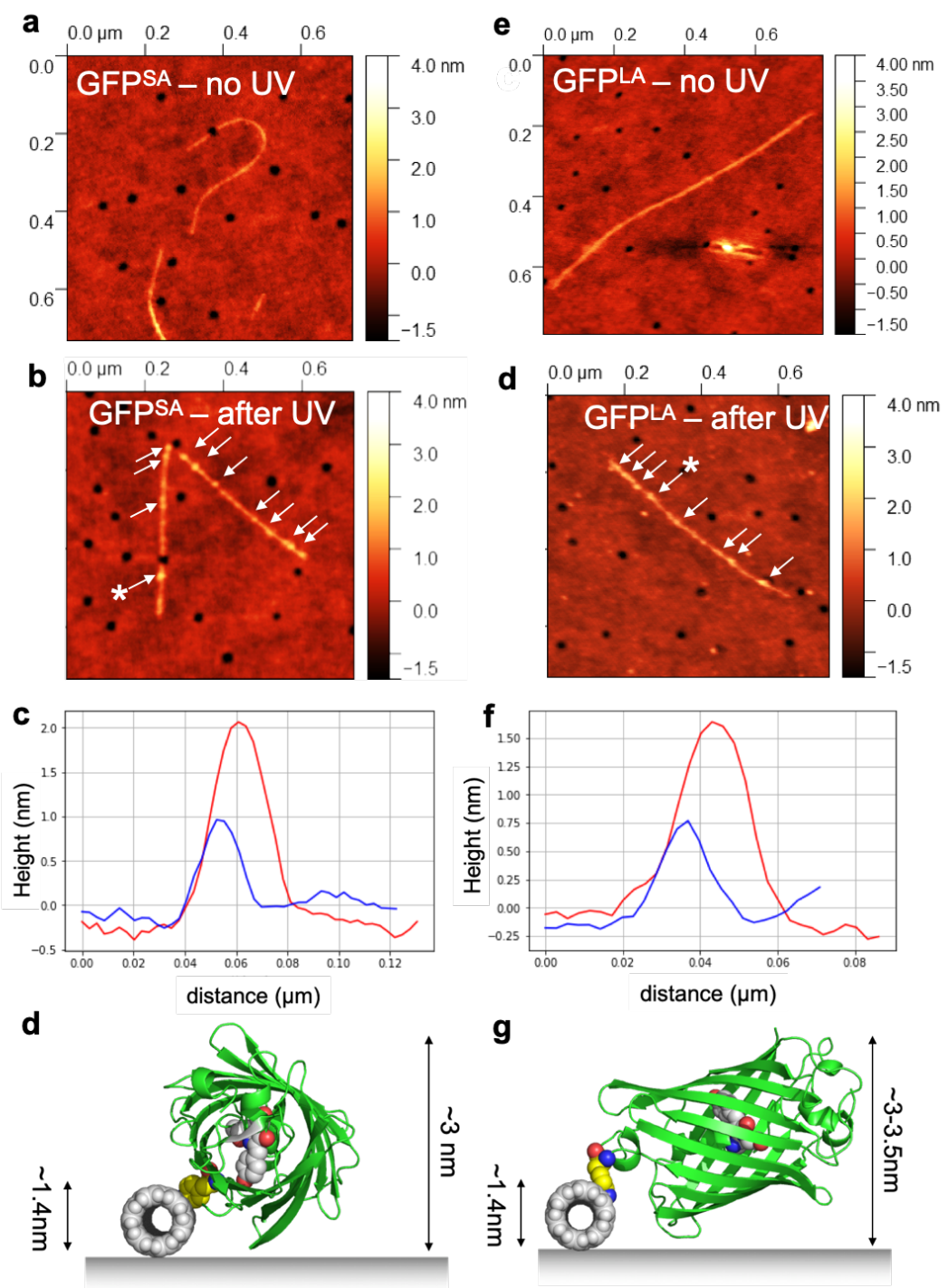

Figure 2. AFM analysis of protein attachment to SWCNTs. GFPSA without (a) and with (b) UV irradiation of samples. (c) Height analysis of single spot (red) and adjacent bare CNT region (blue). Height are measured along a trajectory perpendicular to CNT tube long axis. The measured protein-CNT spot is labelled with * in (a). (d) Schematic representation of a potential GFPSA_CNT interaction orientation. GFPLA without (d) and with (e) UV irradiation of samples. (f) Height analysis of single spot (red) and adjacent bare CNT region (blue). Height are measured along a trajectory perpendicular to CNT tube long axis. The measured GFPLA-CNT spot is labelled with * in (a). (g) Schematic representation of a potential GFPLA-CNT interaction orientation. The full height analysis plots are shown in Figures S1 and S2. 


\section{Broad applicability of genetically encoded direct photochemical attachment of proteins.}

To demonstrate the generality of our approach, we incorporated azF at defined positions in two additional proteins: an enzyme associated with antimicrobial resistance (residue 87 of TEM $\beta$-lactamase $(47,48$ ); Figure 1c) and a metalloprotein (residue 50 of cyt $b_{562}(49)$; Figure $1 d$ ). In addition to their different inherent function to GFP they also sample different structural classes. While GFP is a largely $\beta$-sheet protein, cyt $b_{562}$ is a helical protein that binds a prosthetic group (haem) and $\beta$ lactamase is mixed helical-sheet enzyme. We have previously shown that mutation to residue 50 of cytochrome $b_{562}$ has little effect on function $(31,49,50)$, and that incorporation of azF does not disrupt function (Figure S4a). TEM $\beta$-lactamase azF variant (termed $\mathrm{BL}^{87 \mathrm{azF}}$ ) had the same overall activity as the wild-type $\mathrm{BL}$ indicating that incorporation of the ncAA had little effect on the protein (Figure S5a).

Subsequent UV irradiation did not have a significant functional effect either. This is to be expected as the $\mathrm{BL}^{87 a z F}$ was designed to have little inherent impact on protein function due to its remoteness from the active site (Figure 1c).

Attachment to SWCNTs was assessed by AFM in air (Figure 3 and S4-S5). In the absence of illumination with $305 \mathrm{~nm}$ UV light, bare CNTs were observed after soaking the samples with protein followed by washing indicating no protein had permanently bound. On irradiation with $305 \mathrm{~nm}$ light, spots with heights taller and broader than that of the tubes are clearly visible, indicating successful protein attachment. As for GFP, the height analysis suggests the proteins are smaller than predicted by the over-simplified assumption that the expected height is the sum of the protein plus the CNT height (Figures S4-S5). Taking into consideration that AFM underestimates heights of biomolecules $(45,46)$, we suggest models of binding mode regarding to the position of phenyl azide attachment position with respect to the CNT (Figures S4d and S5d); given the relative position of the phenyl azide group with respect to the rest of the protein and the available circumference around the tube available for nitrene insertion, it is unlikely the proteins occupy a fully upright orientation directly above the nanotube. 

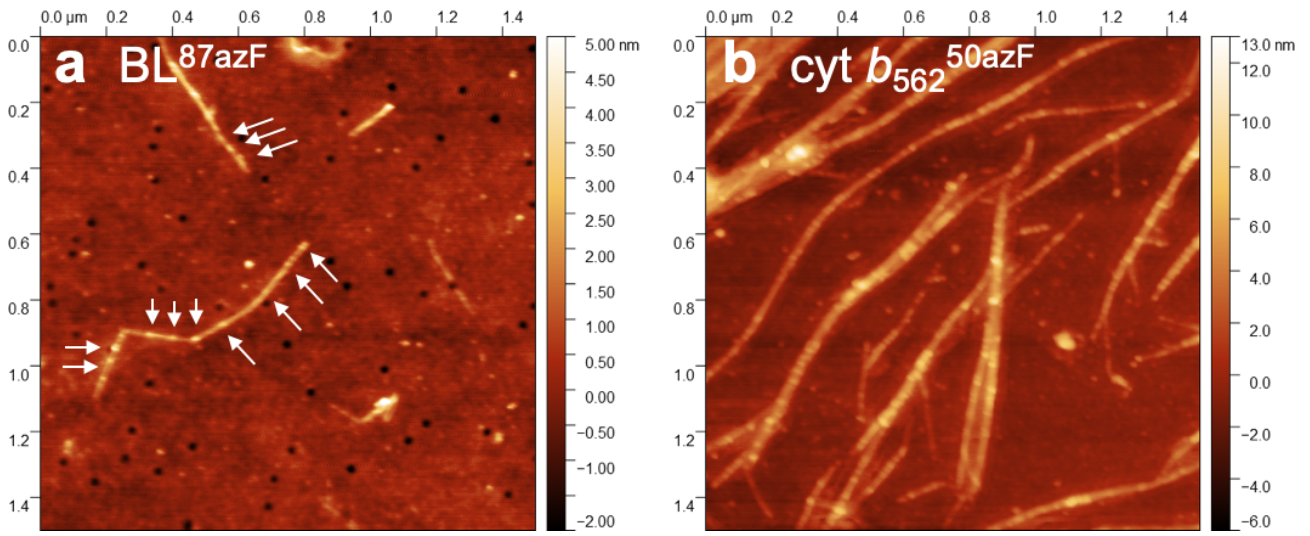

Figure 3. AFM analysis of (a) $\beta$-lactamase (BL) 87azF variant and (b) cytochrome $b_{562}$ (cyt $b_{562}$ ) 50azF variant. Each panel presents a representative AFM image of protein (10 $\mathrm{nM}$ ) deposited on coverslips and then irradiated with $305 \mathrm{~nm}$ UV light. White arrows indicate positions of bound proteins in (a). Height analysis together with control experiments of tubes (no UV exposure prior to washing) are shown in Figures S4 and S5.

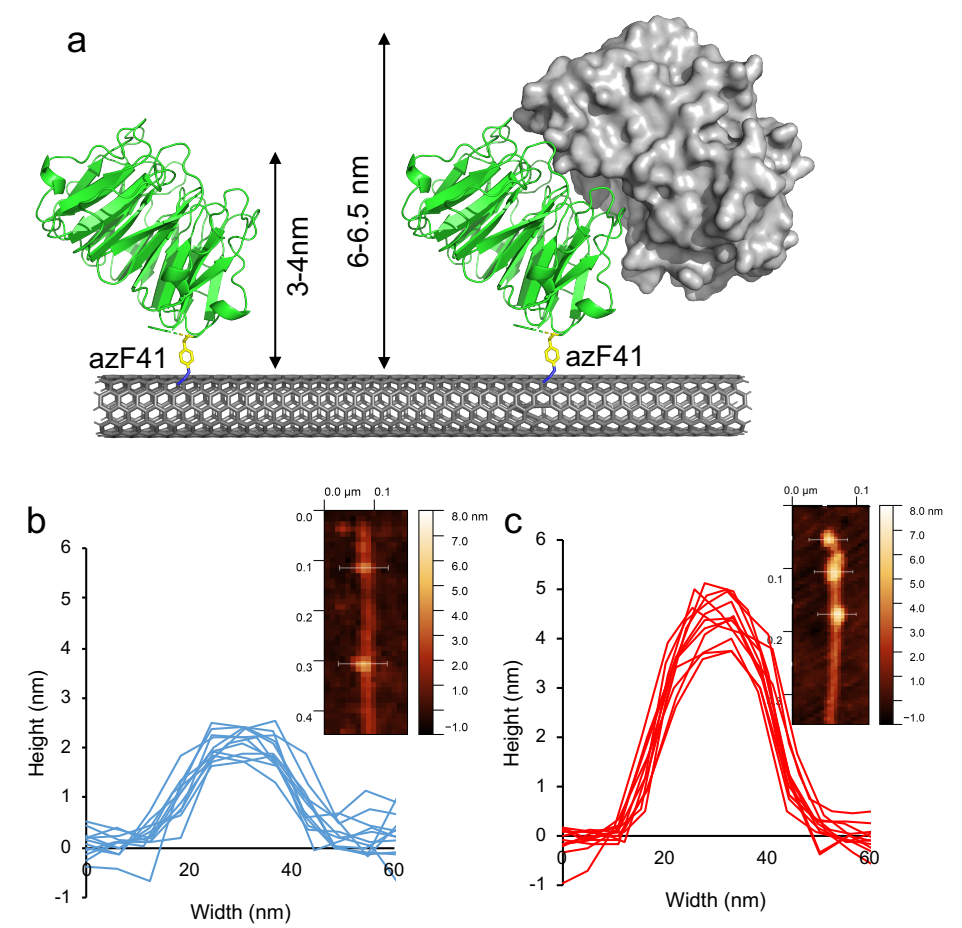

Figure 4. AFM analysis of BBP attachment to SWCNTs. (a) Schematic of BBPSWCNT attachment and subsequent binding to BL. The relative height differences between the BBP and BBP-BL complex are indicated on the diagram based on the crystallographic structure of the complex (PDB 1jtg (51)). (b) Protein height profiles of BBP ${ }^{41 a z F} ; 12$ cross sections were taken across SWCNT-bound proteins and the 
height of bare SWCNT regions was subtracted. The topography of a representive $\mathrm{BBP}^{41 \mathrm{AzF}}$ bound to a SWCNT is shown inset. (c) Protein height profiles $(n=12)$ of $\mathrm{BL}$ bound to BBP ${ }^{41 a z F}$ on SWCNTs; the height of bare SWCNT regions was subtracted. The topography of BBP ${ }^{41 a z F}-B L$ complex bound to SWCNT is shown inset.

\section{Protein complex formation}

The attachment of proteins capable of binding other biomolecules, including other proteins is important for long term applications in biosensing. Here we show that our approach can attach such binding proteins to SWCNTs, and that they retain their binding ability. Given we have shown above that $\mathrm{BL}$ has no inherent ability to bind CNTs (Figure S4), we engineered a $\beta$-lactamase binding protein (52) (BBP, also referred to as BLIP2) to site-specifically attach via an azF incorporated at residue 41 (Figure 4a). BBP is a $\beta$-propeller protein that acts as a "universal antibody" for serine $\beta$-lactamases, one of the most common cause of resistance to antibiotics, due to its ability to bind a wide range of this enzyme class. Residue 41 is close to the $\mathrm{N}$ terminal of BBP and should not interfere with the BL interaction interface nor prevent binding even on attachment to a nanotube (see Figure 4a for a schematic outline of binding).

As with all our tested proteins to date, no permanent binding of BBP was observed in the absence of UV light to CNTs after washing (Figure S6). Illumination with UV led to binding of BBP to the CNTs as evidenced by AFM (Figure 4b). The average observed height of BBP was $2.2 \mathrm{~nm}$. Based on the crystal structure we estimate the height to between 3-4 $\mathrm{nm}$, depending the exact geometry of the interaction. The lower observed height is consistent with our previous measurements. For BBP to bind $\mathrm{BL}$ it needs to be correctly folded and the binding interface accessible. On binding $B L$ the height of the peaks increased by $2.5 \mathrm{~nm}$ (measured height of $4.5 \mathrm{~nm}$ ). This is in keeping with the expect increase in height of $\sim 3 \mathrm{~nm}$ based on the crystal structure of the complex and the known underestimation of protein height by AFM. Thus, our approach will allow the sampling of protein complex formation at fixed and defined orientations with respect to the SWCNT surface through attaching binding proteins at specific sites. 


\section{Influence of SWCNT attachment on GFP single molecule behaviour.}

GFP provides an opportunity to study the effect intimate and direct covalent attachment to SWCNTs has on protein function down to the single molecule level. Fluorescence is sensitive to local changes in environment allowing any potential effects CNT attachment has to be monitored. If SWCNT binding is influencing GFP, changes in fluorescence properties should be observed. Importantly, if GFP is unfolded fluorescence is lost (44). This is not the case here as single molecule fluorescence is observed on attachment (Figure 5). If protein attachment site modulates such communication, we should see differences in fluorescence between the two variants. Steady state bulk fluorescence confirms our previous studies (21) that UV irradiation has little effect on the GFPWT, GFPLA and GFPSA used in this study when the proteins are free in solution (Figure S7).

Total internal reflection fluorescence microscopy (TIRFM) provides an alternative approach to confirm attachment of the GFP azF variants to SWCNTs as well as checking they retain function and to investigate the influence attachment has on fluorescence at single molecule resolution. For both GFPSA and GFPLA, extended fluorescent structures were observed by TIRFM after irradiation with UV light indicating the proteins attached along the length of the SWCNTs and that they retained their function (Figure 5 and S8). Such extended fluorescent structures were absent when using GFPWT under similar conditions (Figure S8). Very few proteins were observed for the azF containing GFP variants in the absence of $305 \mathrm{~nm}$ light (Supporting Movies S1 and S2); those that were detected behaved like free protein with high initial fluorescence followed by quick photobleaching. These observations confirm the AFM data that both azF and irradiation with UV light are required to stability attach our engineered proteins to SWCNTs. 

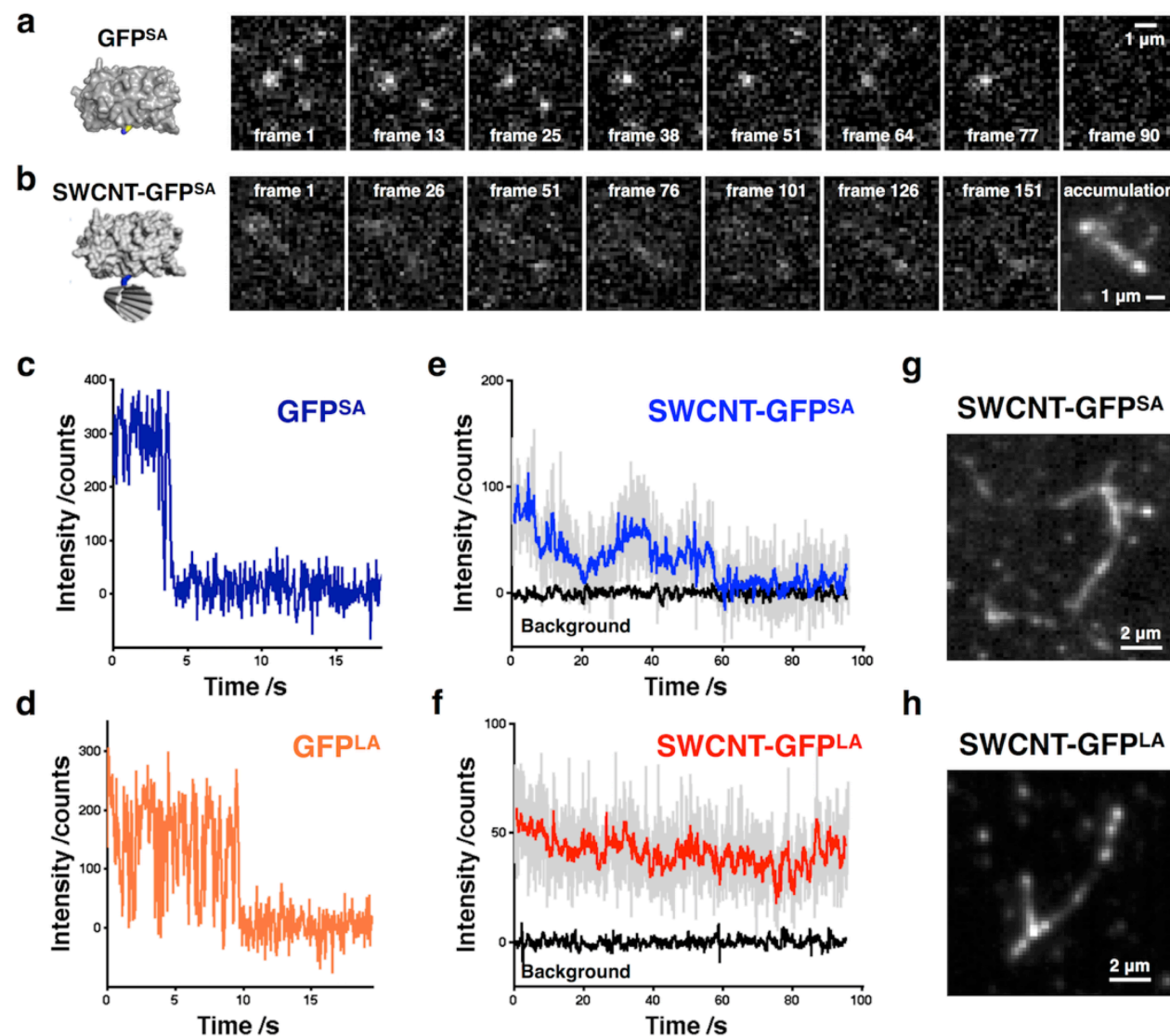

h

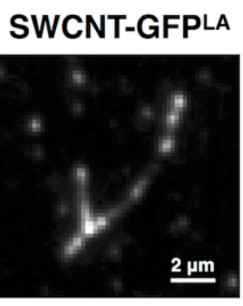

Figure 5. Single molecule imaging of GFPSA and GFPLA free in solution and SWCNT bound. (a-b) TIRF imaging time course of GFPSA free and CNT attached. (a) Single free GFPSA are observed that stochastically photobleach. See also Supporting Movie S3. (b) SWCNT-GFPSA is less bright and undergoes significant intensity fluctuations. Summing frames reveals the linear structure of the decorated SWCNTs. The time course for GFPLA is shown in Supporting Figure 9. See also Supporting Movie S4 (cf) Representative intensity traces of GFPSA and GFPLA free and SWCNT bound (See figure S10 for additional traces). GFPSA and GFPLA free in solution (c-d) and attached to SWCNT (e-f). Attachment alters the photophysics of the fluorescent protein; reducing fluorescence intensity, prolonging photobleaching lifetime and inducing fluctuation. Raw data (grey), Chung Kennedy filtered data (blue/red) and Chung Kennedy filtered background intensity traces are shown) (g-h) GFPSA and GFPLA decorated SWCNTs revealed by TIRF.

Attachment of the GFP variants to SWCNTs has a significant influence on protein fluorescence. The GFP-SWCNTs structures exhibited reduced intensity compared to proteins free in solution and displayed temporal fluctuations in addition to periodic 
on/off blinking (Figure 5). TIRFM imaging (Figure 5-a-b) illustrates the change in fluorescence on GFPSA coupling to SWCNTs. Single spots were observable until they stochastically photobleached for GFPSA free in solution (Figure 5a). In contrast, weaker fluorescence is observed for SWCNT-GFPSA with low intensity spots fluctuating and blinking (Figure $5 \mathrm{~b}$ ). The addition of successive frames reveals the overall GFPAA decorated CNT structure (Figure 5b).

Extraction of intensity traces from individual spots of SWCNT coupled protein (Figure $5 e$ and $5 f$, Figure S10) shows the decrease in fluorescence intensity, the increase in photobleaching lifetime and stochastic fluctuations in intensity, compared to the free protein, suggestive of a dynamic photophysical interaction with the SWCNT. Whilst SWCNT coupled GFPSA eventually photobleaches to background during the course of the experiment, SWCNT-GFPLA, although less bright, is more resistant to bleaching and gives a more consistent output. Both variants exhibit dynamic fluctuations of small magnitudes, rarely showing discrete steps, unlike the free protein counterparts. The observed fluctuations are not readily attributed to a small number of discrete states in our data, suggesting highly dynamic processes, likely the result of continuous dynamic changes in events, possibly energy and/or charge transfer, events between protein and the attached SWCNT.
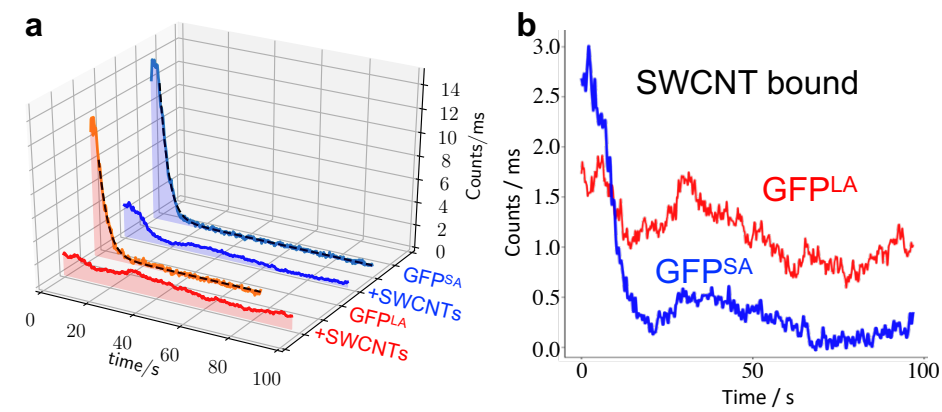

Figure 6. Comparison of ensemble single molecule TIRFM photobleaching kinetics of GFPSA (blue) and GFPLA (red). A reduction in fluorescence intensity and prolongation of photobleaching lifetime is observed for both GFP attachment orientations (a). This change in fluorescence characteristics manifests as different ensemble photobleaching behaviour between GFPSA (blue) and GFPLA (red) attachment (b). SWCNT attachment ensemble decay deviates from the simple exponential decay of the proteins in solution. The data in (b) is an overlay of the data shown in (a) for the proteins attached to SWCNTs. 
Extraction of ensemble decay curves yields exponential decay characteristics for both protein variants in solution. As the single molecule image analysis suggested, GFPLA was slightly less intense compared to GFPSA and had a shorter fluorescence decay time of $3.2 \mathrm{~s}$ versus $3.74 \mathrm{~s}$ (Figure 6a). Attached to SWCNTs the ensemble decay deviates from a simple photobleaching exponential (Figure 6b), indicating more complex behaviour. Globally across the sample, this manifests as an initial decay followed by an apparent recovery phase ( $20 \mathrm{~s})$ which subsequently decays. This behaviour can be better understood in conjunction with the single molecule traces where fluctuation and blinking events are observed. In an ensemble collection, the apparent second phase is most likely due to a population of molecules that are initially in the "off" state that stochastically activate (see supplementary traces in Figure S10). In these ensemble traces SWCNT-GFPSA tends toward zero over the course of the experiment which is consistent with complete photobleaching. Despite being less bright initially, GFPLA decay is slower and does not totally bleach during the experimental acquisition (96 s) suggesting a much longer decay time. This increased resistance to photobleaching is reflected in observations at the single molecule level (Figure 5e-f).

\section{Interaction between protein and SWCNT: importance of attachment site.}

The major changes in fluorescence suggests that there is some degree of interaction between the proteins and the SWCNT. The different fluorescent behaviour between GFPLA and GFPSA indicates that attachment position (with respect to the protein) plays a role in determining this apparent communication. The classic mechanism to explain reduced intensity is energy transfer, in this case from GFP to SWCNT, which can occur when there is a nearby acceptor $(53,54)$. However, simple energy transfer alone does not explain the longer decay times that accompany the lower intensities, which may suggest that photocycling rate as part of the excitation, emission and recovery events is slower. Simultaneous energy transfer events from the multiple proteins on a single SWCNT could also generate feedback to individual GFPs. The attachment of multiple proteins to single SWCNTs may result in multiple simultaneous energy and/or charge transfer events to a single tube creating perturbations and thus feedback to individual fluorescent proteins, resulting in the more continuous, rather than discrete, step-like state behaviour usually observed 
with single molecules. This would be consistent with the small number of discrete step-like events that are observed in our data as originating from SWCNTs with single or low numbers of decorated functional proteins, limiting the influence of simultaneous protein-CNT energy transfer and feedback. The fluorescence behaviour of GFPLA was affected to a greater extent than GFPSA, with the former exhibiting reduced intensity but little photobleaching even after $\sim 90 \mathrm{~s}$ (Figures 4 and $6)$. This is unexpected given the differences in distance and positions between the attachment azF residue and the chromophore functional centre (Figure 1b). Indeed, these observations for SWCNT side-wall attachment differ markedly from recent work concerning end-wall attachment where the largest effect was observed for GFPSA (41), highlighting how different linkage positions in different contexts give rise to different effects. Analysis of the GFP structure with CAVER (55), which detects possible channels including water tunnels, show possible channels between protein and SWCNT (Figure S11). This observation together with previous work on waterbased charge transfer networks in $\operatorname{GFP}(38,56,57)$, suggests that residue 132 is close the exit of an internal water tunnel coupled directly to the chromophore. By coupling to the electron rich pi bond network of the SWCNT side wall close to the tunnel exit may provide a mechanism for the influence of the SWCNT on fluorescence. While the exact nature of coupling between GFP and SWCNT and the associated networks needs to be investigated further, it does not appear to be a simple distance dependent relationship highlighting the potential importance of coupling position in terms of existing bond networks commonly found in proteins.

\section{Conclusion.}

Here we have shown that a protein can be intimately linked at defined single residue positions to the side-wall of SWCNTs in a facile and direct manner using light as the facilitator; genetic incorporation of phenyl azide chemistry is the key and can potentially be applied to any protein of interest (58). Such approaches are required to facilitate consistent fabrication of bionanohybrid devices, especially when single molecule behaviour of proteins is to be utilised. We demonstrate attachment using four different proteins, including a binding protein that has long term potential as a biosensor for detection of biomarkers related to antimicrobial resistance. Attachment of protein to a CNT is not necessarily a benign effect. The observed changes in fluorescence on attachment of GFP to SWCNTs suggests coupling between the two 
systems, with the degree of influence on fluorescence dependent on the protein attachment position and orientation. Thus, defining the protein attachment position not only allows consistent fabrication but allow the effect one has on the other to be adapted to influence to degree of modulation. The latter is especially important given the prevalence of charge transfer in protein biochemistry, including catalysis, signal transduction and energy utilisation.

\section{Methods.}

SWCNT preparation and protein attachment. A solution of single wall carbon nanotubes was prepared from powder (Sigma Aldrich: single walled diameter $0.7-1.4 \mathrm{~nm}$, carbon $>90 \%$, $\geq 77 \%$ carbon as SWCNT). The powder was dispersed into a solution $1 \% \mathrm{w} / \mathrm{v}$ SDS (Sigma Aldrich, molecular biology grade). The solution was sonicated using a probe sonicator in bursts of 10 seconds for five iterations, followed by 30 seconds for three iterations and finally 60 seconds for five iterations. After this treatment the grains of SWCNT can be visually confirmed to have decreased in density, thus implying a good dispersion within the solution. The SWCNT solution was then divided into $750 \mu$ l aliquots and placed into a bench top microfuge for 30 minutes at 14,000rpm. Once a pellet had formed at the bottom of the solution, the supernatant was removed and used for subsequent preparations. The prepared SWCNT solution ( $20 \mu \mathrm{l})$ was drop cast onto the surface of a plasma treated glass coverslip and allowed to dry for 30 minutes. Any solution remaining on the surface was washed away under flowing DI water followed by a 10 second wash with flowing ethanol (Fischer Chemicals, analytical reagent grade). The coverslip was then submerged in ethanol for 1 hour to remove any surfactant or unbound tubes from the surface. Finally, the coverslip was given a final rinse with flowing DI water to remove the ethanol and dried under $\mathrm{N}_{2}$ stream.

The engineering and production of proteins is described in Supporting Methods and is based on procedures described elsewhere(33). In order to bind protein to the carbon nanotubes on the coverslip substrate, low humidity conditions were used to minimise the effect of oxidation. Using a low humidity glove box, the SWCNT samples were placed on a specially designed stage within a small lock box. This allowed the $305 \mathrm{~nm}$ LED mounted in the lid of the lock box to be positioned directly above the sample. Protein solution ( $20 \mu \mathrm{l}$ of $10-100 \mathrm{nM}$ samples in $50 \mathrm{mM}$ Tris $\mathrm{HCl} \mathrm{pH} \mathrm{8)} \mathrm{was} \mathrm{drop} \mathrm{cast} \mathrm{onto} \mathrm{the} \mathrm{surface} \mathrm{of} \mathrm{the} \mathrm{sample} \mathrm{and} \mathrm{the} \mathrm{lock}$ box closed. For illumination experiments, the $305 \mathrm{~nm}$ LED with an intensity of $18 \mathrm{Wm}^{-2}$ was switched on and the sample left for 5 minutes. The sample was then removed from the stage and rigorously washed for 30 seconds under flowing DI water. Control samples were 
included that did not undergo illumination prior to washing. The sample was finally dried and removed from the low humidity for mounting and imaging. To form the $\mathrm{BBP}^{41 \mathrm{azF}}-\mathrm{BL}$ complex on SWCNTs, first BBP ${ }^{41 a z F}$ was attached using the aforementioned method, rinsed and dried, followed by AFM imaging. The BBP ${ }^{41 a z F}-B L$ complex was formed by drop casting $20 \mu \mathrm{l}$ of $100 \mathrm{nM} \mathrm{BL}$ onto the coverslip and left for 5 minutes in dark conditions. Coverslips were rinsed thoroughly with deionised water for 30 seconds and dried in the low humidity conditions, before mounting and imaging.

Single molecule imaging. AFM imaging is described in detail in the Supporting Information. Single molecule TIRF microscopy was performed as followed. Single molecule imaging was performed using a custom built total internal reflection fluorescence (TIRF) microscope based on a Nikon Ti-U inverted microscope and Andor iXon ultra 897 EMCCD camera. Illumination was provided by a Ventus $473 \mathrm{~nm}$ DPSS laser with a power output of $100 \mathrm{~mW}$. Laser coupling into the microscope was achieved via a custom built optical circuit (components were sourced from Thorlabs, Chroma and Semrock) followed by a single mode fibre-optic launch. Laser power at the microscope stage averaged at $5.8 \mu \mathrm{W} / \mu \mathrm{m}^{2}$. The total internal reflection illumination angle was generated using a combination of fibre-optic micropositioning and a high numerical aperture TIRF objective (Nikon, CFI Apochromat TIRF 60X oil, NA1.49). The Excitation and fluorescence emission wavelengths were separated using a dichroic mirror with a 488nm edge (Chroma zt488rdc-xr). Emitted wavelengths were further filtered using a 500nm edge long pass filter (Chroma hhq500lp) and a 525nm band pass filter (Chroma et525/50m). Acquisitions were controlled using the Andor Solis software package. Frame exposure times were set to $60 \mathrm{~ms}$ and an EM gain of 250 was used. Information was saved in Andor's proprietary .sif format to preserve file associated metadata.

SWCNT deposition and protein attachment is similar to that used for AFM imaging. Coverslips used for TIRF imaging were plasma treated to remove contaminants, then coated in $0.01 \mathrm{mg} / \mathrm{ml}$ SWNTs in SDS solution. Samples were then rinsed with deionised water and soaked in ethanol for 1 hour, followed by a rinse under a flow of ethanol and dried in a flow of $\mathrm{N}_{2}$. Protein solutions were deposited on the coverslips whilst they were examined under the microscope. Photo-cross linking to CNTs was also performed on the microscope stage using the $305 \mathrm{~nm}$ LED with an intensity of $18 \mathrm{Wm}^{-2}$ for 5 minutes before rinsing on the stage with deionised water. Protein solutions diluted to single molecule concentrations (as determined by TIRF microscopy), in $50 \mathrm{mM}$ Tris $\mathrm{HCl} \mathrm{pH} 8$ were deposited on the coverslips whilst they were examined under the microscope. Photo-cross linking to CNTs was also performed on the microscope stage using the 305nm LED with an intensity of $18 \mathrm{Wm}-2$ for 5 minutes before rinsing on the microscope stage with deionised water. 
Single molecule imaging data were processed and analysed using ImageJ (59) and Matlab (R2012a) (MathWorks U.S.A.). 32 bit floating point TIFF image stacks were used throughout. The first acquisition frame was removed from all image sequences to account for latency of shutter opening by the camera TTL trigger. All images were processed to normalise for spatial variation in intensity profile of the laser illumination using a reference image look-up of relative spatial illumination intensity mapping the laser illumination created from a Gaussian blurred (20 pixel radius) median z-projection of a fluorescent image stack. The resulting image stack was then corrected for temporal laser intensity fluctuations to minimise the noise in extracted traces. This was achieved by quantifying fluctuations in the global image background and scaling the corresponding frame accordingly, relative to the mean (i.e. this meant dividing each frame by a value, between 0.98 and 1.02 corresponding to the relative laser intensity of any given frame). Practically, this was achieved by removing bright fluorescent spots, defined as any pixel with an intensity greater than 0.05 standard deviations above the median pixel intensity of that frame. Identified pixels were assigned a value equal to the median pixel intensity, effectively erasing them to give a background only image stack. Each frame was scaled relative to the mean intensity of all frames (all pixels) and used to create a temporal lookup table of relative frame to frame laser power fluctuations. This enabled correction of the main image stack. Background counts were subtracted by the pixel-wise subtraction of time averaged median pixel intensity of a background region of interest. Spots were detected in either single frames or projected images using the ImageJ plugin trackmate (60), integrated in the FIJI (61) distribution of ImageJ, prior to frame-wise intensity extraction. The resultant intensity traces were plotted and analysed in Matlab.

For the ensemble measurements, the image files were converted on a frame-by-frame basis to counts/ms, with each frame corresponding to $60 \mathrm{~ms}$. A flat field correction was made by subtracting (in a time dependent manner) background from an appropriate region of the image. The time series was smoothed using a Savitzky-Golay filter (https://scipy.github.io/devdocs/generated/scipy.signal.savgol filter.html). Bright pixels (corresponding the GFP) made by averaging the first 100 frames that were clearly separated from each other were selected and the plot of the average intensity of these selected pixels was generated. To generate the ensemble plot the following number of individual protein associated fluorescent spots were used: 74 for free GFPSA; 25 for GFPSA bound to SWCNTs; 76 for free GFP ${ }^{L A} ; 45$ for GFP ${ }^{L A}$ bound to SWCNTs.

\section{Acknowledgements}


The authors would like to thank the BBSRC (BB/H003746/1 and BB/M000249/1), EPSRC (EP/J015318/1) and Cardiff Synthetic Biology Initiative in association with SynbiCITE for supporting this work. SKT and AB were supported by EPSRC studentships, BJB and REAG by SWBio DTP BBSRV studentships, and HLW with a Cardiff University BBSRC-facing studentship. WDJ is supported by a Wellcome Trust ISSF project grant. We would like to thank the Cardiff School of Biosciences Protein Technology Hub for helping with production and analysis of proteins. The BL (TEM) and BBP (BLIP2) containing plasmid was kindly donated by Prof Tim Palzkill.

\section{References}

(1) De Leo, F., Magistrato, A., and Bonifazi, D. (2015) Interfacing proteins with graphitic nanomaterials: from spontaneous attraction to tailored assemblies. Chem Soc Rev 44, 6916-53.

(2) Wen, J., Xu, Y., Li, H., Lu, A., and Sun, S. (2015) Recent applications of carbon nanomaterials in fluorescence biosensing and bioimaging. Chemical communications 51, 11346-58.

(3) Georgakilas, V., Otyepka, M., Bourlinos, A. B., Chandra, V., Kim, N., Kemp, K. C., Hobza, P., Zboril, R., and Kim, K. S. (2012) Functionalization of graphene: covalent and non-covalent approaches, derivatives and applications. Chem Rev 112, 6156-214.

(4) Peng, X., Chen, J., Misewich, J. A., and Wong, S. S. (2009) Carbon nanotube-nanocrystal heterostructures. Chem Soc Rev 38, 1076-98.

(5) Karousis, N., Tagmatarchis, N., and Tasis, D. (2010) Current progress on the chemical modification of carbon nanotubes. Chem Rev 110, 5366-97.

(6) Mallakpour, S., and Soltanian, S. (2016) Surface functionalization of carbon nanotubes: fabrication and applications. RSC Advances 6, 109916-109935.

(7) Calvaresi, M., and Zerbetto, F. (2013) The devil and holy water: protein and carbon nanotube hybrids. Acc Chem Res 46, 2454-63.

(8) Mehra, N. K., Mishra, V., and Jain, N. K. (2014) A review of ligand tethered surface engineered carbon nanotubes. Biomaterials 35, 1267-83.

(9) Xu, X., Clement, P., Eklof-Osterberg, J., Kelley-Loughnane, N., Moth-Poulsen, K., Chavez, J. L., and Palma, M. (2018) Reconfigurable Carbon Nanotube Multiplexed Sensing Devices. Nano Lett 18, 4130-4135.

(10) Sims, P. C., Moody, I. S., Choi, Y., Dong, C., Iftikhar, M., Corso, B. L., Gul, O. T., Collins, P. G., and Weiss, G. A. (2013) Electronic measurements of singlemolecule catalysis by cAMP-dependent protein kinase A. J Am Chem Soc 135, 7861-8.

(11) Grigoryan, G., Kim, Y. H., Acharya, R., Axelrod, K., Jain, R. M., Willis, L., Drndic, M., Kikkawa, J. M., and DeGrado, W. F. (2011) Computational design of virus-like protein assemblies on carbon nanotube surfaces. Science 332, 1071-6.

(12) Choi, Y., Moody, I. S., Sims, P. C., Hunt, S. R., Corso, B. L., Perez, I., Weiss, G. A., and Collins, P. G. (2012) Single-molecule lysozyme dynamics monitored by an electronic circuit. Science 335, 319-24. 
(13) Goldsmith, B. R., Mitala, J. J., Josue, J., Castro, A., Lerner, M. B., Bayburt, T. H., Khamis, S. M., Jones, R. A., Brand, J. G., Sligar, S. G., Luetje, C. W., Gelperin, A., Rhodes, P. A., Discher, B. M., and Johnson, A. T. (2011) Biomimetic chemical sensors using nanoelectronic readout of olfactory receptor proteins. ACS Nano 5, 5408-16.

(14) Wang, S., Humphreys, E. S., Chung, S. Y., Delduco, D. F., Lustig, S. R., Wang, H., Parker, K. N., Rizzo, N. W., Subramoney, S., Chiang, Y. M., and Jagota, A. (2003) Peptides with selective affinity for carbon nanotubes. Nat Mater 2, 196-200.

(15) Balasubramanian, K., and Burghard, M. (2005) Chemically functionalized carbon nanotubes. Small 1, 180-92.

(16) Marchesan, S., and Prato, M. (2015) Under the lens: carbon nanotube and protein interaction at the nanoscale. Chemical communications 51, 4347-59.

(17) Steen Redeker, E., Ta, D. T., Cortens, D., Billen, B., Guedens, W., and Adriaensens, P. (2013) Protein engineering for directed immobilization. Bioconjug Chem 24, 1761-77.

(18) Brase, S., Gil, C., Knepper, K., and Zimmermann, V. (2005) Organic azides: an exploding diversity of a unique class of compounds. Angew Chem Int Ed Engl 44, 5188-240.

(19) Costa, R. D., Fernandez-Luna, V., and Coto, P. (2017) When Fluorescent Proteins Meet White Light-Emitting Diodes. Angew Chem Int Ed Engl.

(20) Chin, J., Santoro, S., Martin, A., King, D., Wang, L., and Schultz, P. (2002) Addition of p-azido-L-phenylalanine to the genetic code of Escherichia coli. $J$ Am Chem Soc 124, 9026-7.

(21) Reddington, S. C., Rizkallah, P. J., Watson, P. D., Pearson, R., Tippmann, E. M., and Jones, D. D. (2013) Different photochemical events of a genetically encoded phenyl azide define and modulate GFP fluorescence. Angew Chem Int Ed Engl 52, 5974-7.

(22) Chin, J. W. (2014) Expanding and reprogramming the genetic code of cells and animals. Annu Rev Biochem 83, 379-408.

(23) Wang, F., Niu, W., Guo, J., and Schultz, P. G. (2012) Unnatural amino acid mutagenesis of fluorescent proteins. Angew Chem Int Ed Engl 51, 10132-5.

(24) Pastine, S. J., Okawa, D., Kessler, B., Rolandi, M., Llorente, M., Zettl, A., and Frechet, J. M. (2008) A facile and patternable method for the surface modification of carbon nanotube forests using perfluoroarylazides. J Am Chem Soc 130, 4238-9.

(25) Liu, L. H., and Yan, M. (2010) Perfluorophenyl azides: new applications in surface functionalization and nanomaterial synthesis. Acc Chem Res 43, 1434-43.

(26) Han, J., and Gao, C. (2010) Functionalization of carbon nanotubes and other nanocarbons by azide chemistry Nano-Micro Letters 2, 213-226.

(27) Hartley, A. M., Worthy, H. L., Reddington, S. C., Rizkallah, P. J., and Jones, D. D. (2016) Molecular basis for functional switching of GFP by two disparate non-native post-translational modifications of a phenyl azide reaction handle. Chemical Science.

(28) Reddington, S. C., Driezis, S., Hartley, A. M., Watson, P. D., Rizkallah, P. J., and Jones, D. D. (2015) Genetically encoded phenyl azide photochemistry drives positive and negative functional modulation of a red fluorescent protein. RSC Advances 5, 77734-77738. 
(29) Morris, J. L., Reddington, S. C., Murphy, D. M., Jones, D. D., Platts, J. A., and Tippmann, E. M. (2013) Aryl azide photochemistry in defined protein environments. Org Lett 15, 728-31.

(30) Strom, T. A., Dillon, E. P., Hamilton, C. E., and Barron, A. R. (2010) Nitrene addition to exfoliated graphene: a one-step route to highly functionalized graphene. Chemical communications 46, 4097-9.

(31) Zaki, A. J., Hartley, A., Reddington, S. C., Thomas, S. K., Watson, P., Hayes, A., Moskalenko, A. V., Craciun, M. F., Macdonald, J. E., Jones, D. D., and Elliott, M. (2018) Defined covalent assembly of protein molecules on graphene using a genetically encoded photochemical reaction handle. RSC Advances 8, 5768-5775.

(32) Pedelacq, J. D., Cabantous, S., Tran, T., Terwilliger, T. C., and Waldo, G. S. (2006) Engineering and characterization of a superfolder green fluorescent protein. Nat Biotechnol 24, 79-88.

(33) Reddington, S. C., Tippmann, E. M., and Jones, D. D. (2012) Residue choice defines efficiency and influence of bioorthogonal protein modification via genetically encoded strain promoted Click chemistry. Chemical communications 48, 8419-21.

(34) Korpany, K. V., Langat, P., Kim, D. M., Edelman, N., Cooper, D. R., Nadeau, J., and Blum, A. S. (2012) Conductance switching in the photoswitchable protein Dronpa. J Am Chem Soc 134, 16119-22.

(35) Fernandez-Luna, V., Coto, P. B., and Costa, R. D. (2018) When Fluorescent Proteins Meet White Light-Emitting Diodes. Angew Chem Int Ed Engl 57, 8826-8836.

(36) Dietrich, C. P., Steude, A., Tropf, L., Schubert, M., Kronenberg, N. M., Ostermann, K., Hofling, S., and Gather, M. C. (2016) An exciton-polariton laser based on biologically produced fluorescent protein. Sci Adv 2, e1600666.

(37) Zajac, J. M., Schubert, M., Roland, T., Keum, C. M., Samuel, I. D. W., and Gather, M. C. (2018) Time-Resolved Studies of Energy Transfer in Thin Films of

Green and Red Fluorescent Proteins. Advanced Functional Materials 28, 1706300.

(38) Shinobu, A., Palm, G. J., Schierbeek, A. J., and Agmon, N. (2010) Visualizing proton antenna in a high-resolution green fluorescent protein structure. J Am Chem Soc 132, 11093-102.

(39) van Thor, J. J. (2009) Photoreactions and dynamics of the green fluorescent protein. Chem Soc Rev 38, 2935-50.

(40) Acharya, A., Bogdanov, A. M., Grigorenko, B. L., Bravaya, K. B., Nemukhin, A. V., Lukyanov, K. A., and Krylov, A. I. (2017) Photoinduced Chemistry in Fluorescent Proteins: Curse or Blessing? Chem Rev 117, 758-795.

(41) Freeley, M., Worthy, H. L., Ahmed, R., Bowen, B., Watkins, D., Macdonald, J. E., Zheng, M., Jones, D. D., and Palma, M. (2017) Site-Specific One-to-One Click Coupling of Single Proteins to Individual Carbon Nanotubes: A SingleMolecule Approach. J Am Chem Soc 139, 17834-17840.

(42) Remington, S. J. (2006) Fluorescent proteins: maturation, photochemistry and photophysics. Curr Opin Struct Biol 16, 714-21.

(43) Tsien, R. Y. (1998) The green fluorescent protein. Annu Rev Biochem 67, 509-44. 
(44) Andrews, B. T., Schoenfish, A. R., Roy, M., Waldo, G., and Jennings, P. A. (2007) The rough energy landscape of superfolder GFP is linked to the chromophore. J Mol Biol 373, 476-90.

(45) Fuentes-Perez, M. E., Dillingham, M. S., and Moreno-Herrero, F. (2013) AFM volumetric methods for the characterization of proteins and nucleic acids. Methods 60, 113-21.

(46) Santos, S., Barcons, V., Christenson, H. K., Font, J., and Thomson, N. H. (2011) The intrinsic resolution limit in the atomic force microscope: implications for heights of nano-scale features. PLoS One 6, e23821.

(47) Hartley, A. M., Zaki, A. J., McGarrity, A. R., Robert-Ansart, C., Moskalenko, A. V., Jones, G. F., Craciun, M. F., Russo, S., Elliott, M., Macdonald, J. E., and Jones, D. D. (2015) Functional modulation and directed assembly of an enzyme through designed non-natural post-translation modification. Chemical Science.

(48) Marciano, D. C., Pennington, J. M., Wang, X., Wang, J., Chen, Y., Thomas, V. L., Shoichet, B. K., and Palzkill, T. (2008) Genetic and structural characterization of an L201P global suppressor substitution in TEM-1 betalactamase. J Mol Biol 384, 151-64.

(49) Della Pia, E. A., Chi, Q., Jones, D. D., Macdonald, J. E., Ulstrup, J., and Elliott, M. (2011) Single-molecule mapping of long-range electron transport for a cytochrome b(562) variant. Nano letters 11, 176-82.

(50) Della Pia, E. A., Macdonald, J. E., Elliott, M., and Jones, D. D. (2012) Direct binding of a redox protein for single-molecule electron transfer measurements. Small 8, 2341-4.

(51) Lim, D., Park, H. U., De Castro, L., Kang, S. G., Lee, H. S., Jensen, S., Lee, K. J., and Strynadka, N. C. (2001) Crystal structure and kinetic analysis of beta-lactamase inhibitor protein-II in complex with TEM-1 beta-lactamase. Nat Struct Biol 8, 848-52.

(52) Fryszczyn, B. G., Adamski, C. J., Brown, N. G., Rice, K., Huang, W., and Palzkill, T. (2014) Role of beta-lactamase residues in a common interface for binding the structurally unrelated inhibitory proteins BLIP and BLIP-II. Protein Sci 23, 1235-46.

(53) Arpino, J. A., Czapinska, H., Piasecka, A., Edwards, W. R., Barker, P., Gajda, M. J., Bochtler, M., and Jones, D. D. (2012) Structural basis for efficient chromophore communication and energy transfer in a constructed didomain protein scaffold. J Am Chem Soc 134, 13632-40.

(54) Ganesan, S., Ameer-Beg, S. M., Ng, T. T., Vojnovic, B., and Wouters, F. S. (2006) A dark yellow fluorescent protein (YFP)-based Resonance EnergyAccepting Chromoprotein (REACh) for Forster resonance energy transfer with GFP. Proc Natl Acad Sci U S A 103, 4089-94.

(55) Chovancova, E., Pavelka, A., Benes, P., Strnad, O., Brezovsky, J., Kozlikova, B., Gora, A., Sustr, V., KIvana, M., Medek, P., Biedermannova, L., Sochor, J., and Damborsky, J. (2012) CAVER 3.0: a tool for the analysis of transport pathways in dynamic protein structures. PLoS Comput Biol 8, e1002708.

(56) Agmon, N. (2005) Proton pathways in green fluorescence protein. Biophys $J$ 88, 2452-61.

(57) Shinobu, A., and Agmon, N. (2017) Proton Wire Dynamics in the Green Fluorescent Protein. J Chem Theory Comput 13, 353-369. 
(58) Reddington, S., Watson, P., Rizkallah, P., Tippmann, E., and Jones, D. D. (2013) Genetically encoding phenyl azide chemistry: new uses and ideas for classical biochemistry. Biochemical Society transactions 41, 1177-82.

(59) Schneider, C. A., Rasband, W. S., and Eliceiri, K. W. (2012) NIH Image to ImageJ: 25 years of image analysis. Nat Methods 9, 671-5.

(60) Jaqaman, K., Loerke, D., Mettlen, M., Kuwata, H., Grinstein, S., Schmid, S. L., and Danuser, G. (2008) Robust single-particle tracking in live-cell time-lapse sequences. Nat Methods 5, 695-702.

(61) Schindelin, J., Arganda-Carreras, I., Frise, E., Kaynig, V., Longair, M., Pietzsch, T., Preibisch, S., Rueden, C., Saalfeld, S., Schmid, B., Tinevez, J. Y., White, D. J., Hartenstein, V., Eliceiri, K., Tomancak, P., and Cardona, A. (2012) Fiji: an open-source platform for biological-image analysis. Nat Methods 9, 676-82. 